\title{
Synthesis and Mesomorphic Properties of New Fluorinated Schiff Base Liquid Crystals
}

\author{
Sie-Tiong Ha and Teck-Leong Lee \\ Department of Chemical Science, Universiti Tunku Abdul Rahman, Jln Universiti, Bandar Barat, 31900 Kampar, Perak, Malaysia \\ Correspondence should be addressed to Sie-Tiong Ha; hast_utar@yahoo.com
}

Received 10 September 2013; Accepted 21 October 2013; Published 16 January 2014

Academic Editors: S. X. Dou, H. Higashimura, V. Ji, and B. Li

Copyright ( 2014 S.-T. Ha and T.-L. Lee. This is an open access article distributed under the Creative Commons Attribution License, which permits unrestricted use, distribution, and reproduction in any medium, provided the original work is properly cited.

\begin{abstract}
Two new compounds, 4-alkanoyloxybenzylidene-4' -fluoroaniline and 4-fluorobenzylidene- $4^{\prime}$-n-alkanoyloxyaniline comprising a terminal fluorosubstituent were studied. The fluoro substituent contributes to the molecular polarizability, thus affecting intermolecular interactions and hence resulting in smectic mesomorphism. The mesomorphic properties were studied using differential scanning calorimetry and polarizing optical microscopy techniques. The mesomorphic properties of compounds studied are strongly dependent on the orientation of the imine $(\mathrm{CH}=\mathrm{N})$ linkage. The former Schiff base exhibited smectic A phase whereas the latter compound did not display any mesophase. Reversed imine linkage has caused depression of mesomorphic property in the compound studied (4-fluorobenzylidene- $4^{\prime}$-n-alkanoyloxyaniline). The mesomorphic properties of the present compounds were compared with other structurally related series to establish the chemical structure-mesomorphic property relationship.
\end{abstract}

\section{Introduction}

Liquid crystals (LCs) have many practical applications in scientific and technological areas, in particular as display devices, OLEDs, anisotropic networks, photoconductors, and semiconductor materials [1-3]. Strong demand of new LCs for applications has led to the synthesis of wide range of mesogens, in particular, thermotropic liquid crystals $[4,5]$. Most thermotropic liquid crystals belonged to the calamitic molecules which have a rigid core that consisted of two or more phenyl rings and one or more flexible terminal alkyl chains. Schiff base is one of the most well-known linking groups used in connecting the rigid core groups. Although it provides a stepped core structure, it maintains molecular linearity, therefore providing better stability and inducing formation of mesophase $[6,7]$. Numerous works have been reported in this area ever since the discovery of MBBA which exhibited room temperature nematic phase [8]. Recently, ester type of Schiff bases has received considerable attentions owing to their interesting properties and substantial temperature range [9-18].

Structure-property relationship is essential knowledge for molecular modifications of the synthesis of new mesogens with targeted properties and future practical applications [6]. Typical terminal substituents exhibiting LC properties are those with electronegative atoms, such as halogens. Halogens are polar substituents possessing strong dipole moments, thus having the ability to promote mesomorphic properties [1921]. The increased dipole moment enhances the stability of the lattice and melting temperatures [7]. As the ionic radius of the terminal substituent increases, the molecules tend to orientate in a parallel arrangement [22]. Thus, smectic polymorphism is not unusual for mesogens with a terminal halogen substituent, and this has been frequently observed as the length of the alkyl or alkoxy chains increases [20,23]. However, the present compound with fluoro substituent exhibited single mesophase.

In this continuation study, we aim to change the orientation of imine linkage on the existing system and to study the influence of reversed imine linkage on mesomorphism of Schiff bases. The involved compounds are 4-alkanoyloxybenzylidene- $4^{\prime}$-fluoroaniline and 4 -fluorobenzylidene- $4^{\prime}$-nalkanoyloxyaniline (Figure 1). FT-IR, ${ }^{1} \mathrm{H}$ and ${ }^{13} \mathrm{C}$ NMR, EIMS, and elemental analysis were employed to elucidate the molecular structure of the title compound whereas the liquid 


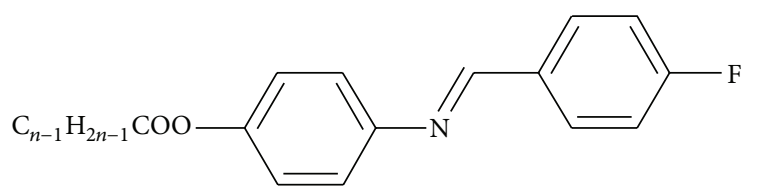

Where $n=8,12$

8FBAA: heating Cr 75.8(24) I; cooling I 25.5(20) Cr 12FBAA: heating Cr 89.7(52) I; cooling I 62.6(51) Cr

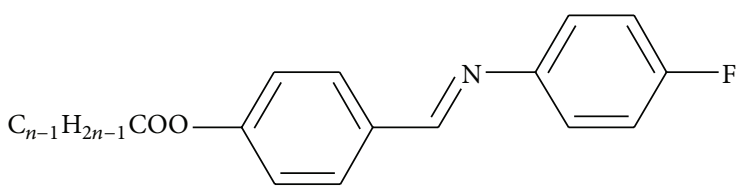

Where $n=8,12$

8ABFA: heating Cr 65.6(26) I; cooling I 42.4(3.9) SmA 27.2(21) Cr 12ABFA: heating Cr 80.5(53) I; cooling 74.1(7.4) SmA 49.6(43) Cr

FIGURE 1: Molecular structure and phase transition temperature $\left({ }^{\circ} \mathrm{C}\right)$ of fluorinated liquid crystals. Note: associated enthalpy changes $\left(\Delta \mathrm{H}, \mathrm{kJ} \mathrm{mol}^{-1}\right)$ are expressed in parentheses.

crystal properties were determined by DSC and POM analysis. In spite of the structural similarity, the smectic nature of the series is quite different. In addition, the relationship between the molecular structure and liquid crystal properties (see Table 1) is also discussed in this paper.

\section{Experimental}

Octanoic acid, dodecanoic acid, 4-fluorobenzaldehyde, 4-aminophenol, 4-fluoroaniline, 4-hydroxybenzaldehyde, 4-dimethylaminopyridine, and N,N'-dicyclohexylcarbodiimide were of analytical grade and used without further purification. The intermediate and title compounds were prepared according to previously reported methods $[10,11]$.

Electron ionization mass spectrum (EI-MS) was recorded using a Finnigan MAT95XL-T mass spectrometer operating at $70 \mathrm{eV}$ ionizing energy. Samples were introduced using a direct inlet system with a source temperature of $200^{\circ} \mathrm{C}$. FT-IR data were acquired with a Perkin Elmer 2000-FTIR spectrophotometer in the frequency range of 4000-400 $\mathrm{cm}^{-1}$ with samples embedded in $\mathrm{KBr}$ pellets. ${ }^{1} \mathrm{H}$ and ${ }^{13} \mathrm{C} \mathrm{NMR}$ spectra were recorded in $\mathrm{CDCl}_{3}$ using a Bruker Avance $300 \mathrm{MHz}$ NMR Spectrometer with TMS as the internal standard. Thin layer chromatography analyses were carried out using aluminum backed silica gel plates (Merck $60 \mathrm{~F}_{254}$ ) and were examined under shortwave UV light.

The phase transition temperatures were measured using a Mettler Toledo DSC823 differential scanning calorimeter (DSC) at a scanning rate of $10^{\circ} \mathrm{C} \mathrm{min}^{-1}$. Liquid crystalline properties were investigated by Carl Zeiss polarizing optical microscope attached to a Linkam Hotstage. The texture of the compounds was observed using polarized light with crossed polarizers; the sample being prepared as a thin film sandwiched between a glass slide and a cover. A video camera (Video Master coomo20P) was installed on the polarizing
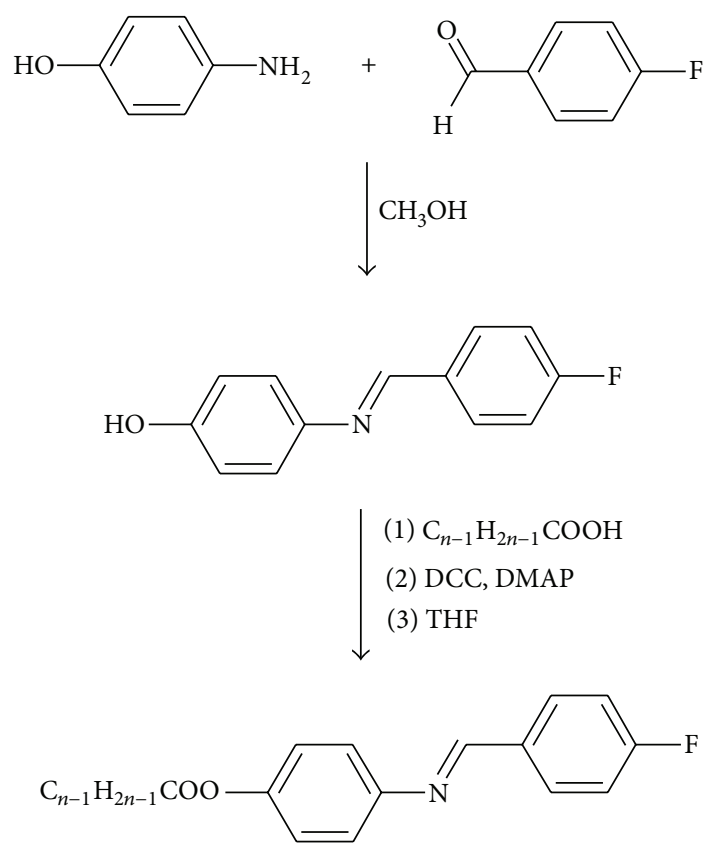

SCHEME 1: Synthetic route towards the formation title compound.

microscope and coupled to a video capture card (Video Master coomo600), allowing real-time video capture and image saving.

2.1. Synthesis of 4-Fluorobenzylidene-4' - hydroxyaniline (OHF$B A A)$. Equal amounts (5 mmol) of 4-fluorobenzaldehyde and 4-aminophenol, along with $30 \mathrm{~mL}$ of methanol, were refluxed for three hours. The mixture was cooled to room temperature and filtered. The yellow product was washed with methanol.

2.2. Synthesis of 4-Fluorobenzylidene-4'-n-dodecanoyloxyaniline (12FBAA). OHFBAA (4 mmol), $3 \mathrm{mmol}$ of dodecanoic acid, DMAP $(0.6 \mathrm{mmol})$, and DCC $(3 \mathrm{mmol})$ were mixed and stirred at room temperature for six hours in appropriate amount of THF (see Scheme 1). Solvent of reaction mixture was removed by evaporation till dryness. Precipitate obtained was recrystallized several times with hexane and ethanol whereupon pure compound was isolated.

EI-MS, IR, and ${ }^{1} \mathrm{H}$ and ${ }^{13} \mathrm{C}$ NMR data of the representative compound 12FBAA are given as follows.

IR (KBr) $v_{\max } \mathrm{cm}^{-1} 2954,2915,2849$ (C-H aliphatic), 1753 (C=O ester), $1628(\mathrm{C}=\mathrm{N}), 1203,1094$ (C-O ester). ${ }^{1} \mathrm{H}$ NMR $\left(300 \mathrm{MHz}_{\mathrm{CDCl}}\right): \delta / \mathrm{ppm} 0.9\left(\mathrm{t}, 3 \mathrm{H}, \mathrm{CH}_{3}-\right), 1.3-1.5(\mathrm{~m}, 16 \mathrm{H}$, $\left.\mathrm{CH}_{3}-\left(\mathrm{CH}_{2}\right)_{8}-\right), 1.8\left(\mathrm{~m}, 2 \mathrm{H},-\mathrm{CH}_{2}-\mathrm{CH}_{2}-\mathrm{COO}-\right), 2.6(\mathrm{t}, 2 \mathrm{H}$, - $\underline{\mathrm{CH}}_{2}-\mathrm{COO}-$ ), 7.1-7.3 (m, 6H, $\mathrm{Ar}-\underline{\mathrm{H}}$ ), 7.9 (m, 2H, Ar- $\underline{\mathrm{H}}$ ), 8.4 $(\mathrm{s}, \overline{\mathrm{H}},-\mathrm{CH}=\mathrm{N}-) .{ }^{13} \mathrm{C} \mathrm{NMR}\left(100 \mathrm{MHz}, \mathrm{CDCl}_{3}\right): \delta / \mathrm{ppm} 172.39$ ( $\mathrm{C}=\mathrm{O}$ ester, $) 158.82(-\mathrm{C}=\mathrm{N}-), 149.34,148.96,132.52,130.85$, $122.22,121.69,116.08,115.79$ for aromatic carbons, 34.42 $\left(-\mathrm{COO}-\mathrm{CH}_{2}-\right)$, 31.90 (-COO- $\left.\mathrm{CH}_{2}-\mathrm{CH}_{2}-\right)$, 29.59, 29.45, $29.32,29.25,29.12$ for methylene carbons $\left[-\left(\underline{\mathrm{CH}_{2}}\right)_{6} \mathrm{CH}_{2}\right.$ $\mathrm{CH}_{2} \mathrm{CH}_{3}$ ], $24.96\left(-\mathrm{CH}_{2} \mathrm{CH}_{2} \mathrm{CH}_{3}\right), 22.67\left(-\mathrm{CH}_{2} \mathrm{CH}_{3}\right), 14.09$ $\left(-\mathrm{CH}_{3}\right)$. EI-MS $m / z$ (rel. int. \%): 397(3) (M) ${ }^{+}, 215(100)$. 
TABLE 1: Mesomorphic data of structurally related compounds.

Compound

$\mathrm{Cl}-\mathrm{ABOH}[27]$

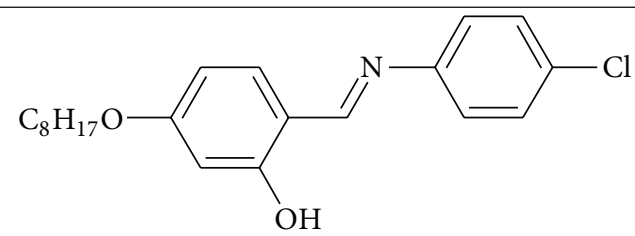

Cr 67 SmA 123 I

$\mathrm{Br}-\mathrm{ABOH}[27]$

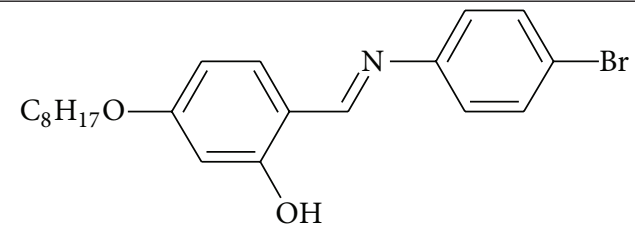

Cr 73 SmA 132 I

I-ABOH [27]

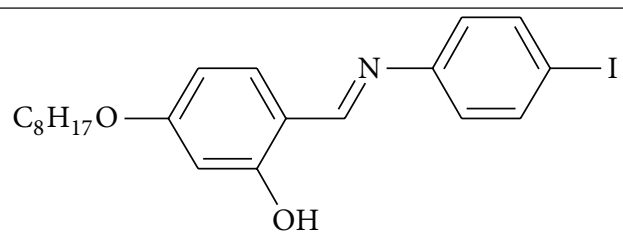

8FBAA<smiles>CCCCCCC(=O)Oc1ccc(/N=C/c2ccc(F)cc2)cc1</smiles>

Cr 76 I

8ABFA<smiles>CCCCOc1c#cc(/C=N/c2ccc(F)cc2)cc1</smiles>

$\operatorname{Cr}(42.4)^{*} \operatorname{SmA} 66 \mathrm{I}$

$8 \mathrm{BACl}[28]$<smiles>CCCCOc1ccc(/C=N/c2ccc(Cl)cc2)cc1</smiles>

$8 \mathrm{BABr}[29]$<smiles>CCCCCC(=O)Oc1ccc(/C=N/c2ccc(Br)cc2)cc1</smiles> 
TABle 1: Continued.

Compound

()$^{*}$ indicates monotropic phase.

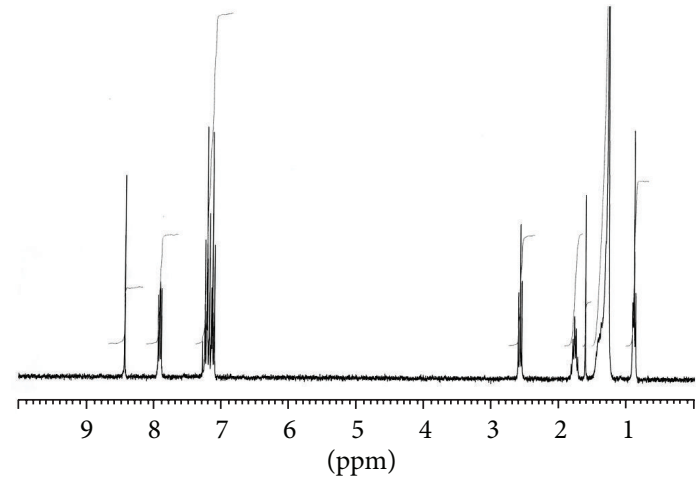

FIGURE 2: ${ }^{1} \mathrm{H}$ NMR spectrum of 12FBAA.

\section{Results and Discussion}

Structural identification of 12FBAA was carried out by using spectroscopic techniques (IR, ${ }^{1} \mathrm{H}$ and ${ }^{13} \mathrm{C}$ NMR, and EI-MS). Molecular ion peak at $m / z 397$ suggested molecular formula of $\mathrm{C}_{25} \mathrm{H}_{32} \mathrm{FNO}_{2}$, which supported the proposed structure of 12FBAA.

3.1. FTIR, ${ }^{1} H$ NMR, and ${ }^{13} C$ NMR Spectral Studies. Based on FT-IR spectrum of 12FBAA, absorption peak at 2954, 2915, and $2849 \mathrm{~cm}^{-1}$ can be assigned to aliphatic $\left(\mathrm{CH}_{3}\right.$ and $\left.\mathrm{CH}_{2}\right)$ groups. Three absorption peaks due to the ester group were observed at 1753, 1203, and $1094 \mathrm{~cm}^{-1}$. Absorption peak of imine $(C=N)$ group was observed at $1622 \mathrm{~cm}^{-1}$. In the ${ }^{1} \mathrm{H}$ NMR spectrum (Figure 2), two triplets at $\delta=0.9 \mathrm{ppm}$ and $\delta=$ $2.6 \mathrm{ppm}$ were, respectively, ascribed to the methyl and methylene protons $\left(-\mathrm{CH}_{2} \mathrm{COO}-\mathrm{Ar}\right)$, while the multiplet between $\delta=1.3-1.5 \mathrm{ppm}$ was assigned to the methylene protons of the long alkyl chain $\left\{-\left(\mathrm{CH}_{2}\right)_{8}-\right\}$. The multiplet signals between $\delta=7.1-7.9 \mathrm{ppm}$ were indicative of the aromatic protons. The singlet observed at the most downfield region, $\delta=8.4 \mathrm{ppm}$, supported the presence of the imine linking group [18]. The molecular structure of 12FBAA was further verified by using ${ }^{13} \mathrm{C}$ NMR spectroscopy (Figure 3). The peak at $\delta=14.09 \mathrm{ppm}$ was attributed to the methyl carbon while the peaks between $\delta=22.67-34.42$ ppm represented the methylene carbons of the long alkyl chain. Twelve aromatic carbons are resonated between $\delta=115.79-149.34 \mathrm{ppm}$. The peaks at $\delta=158.82 \mathrm{ppm}$ and $\delta=172.39$ ppm confirmed the presence of the azomethine carbon and the carbonyl group in the molecule.
3.2. Mesomorphic Properties. 12ABFA exhibited interesting thermotropic properties and its melting behavior was carefully monitored by POM during both heating and cooling scans. The results from the POM observation were verified by the DSC measurements. Optical photomicrograph and DSC thermogram of 12ABFA are shown in Figures 4 and 5 as representative illustration. The transition temperatures, enthalpy changes, and phase sequences are summarized in Figure 1. Phase identification was based on the optical textures, and the magnitude of isotropization on enthalpies is consistent with the assignment of each mesophase type, using the classification systems reported by Sackmann and Demus [24] and Gray and Goodby [25]. Under POM, focal conic fanshaped and homeotropic textures of a smectic A (SmA) phase was observed during the cooling cycle (Figure 4).

3.3. Influence of Alkyl Chain Length on Mesomorphic Properties. Members from the $n \mathrm{ABFA}$ series are monotropic smectogen whereby mesophase (SmA) was only observed during the cooling scan. In monotropic mesogens, the melting points were always equal to or higher than the clearing points, hence exhibiting supercooling properties. As for $n$ FBAA series, both members did not possess mesomorphic properties.

As the alkyl chain length increases, the melting point shows ascending trend. It has been reported that melting point within a homologous series vary depending on molecular mass wherein molecule with lower mass possesses lower melting point in comparison to member with higher mass [6]. Melting point increases when polarizability of the compounds within the same series increases.

3.4. Structure-Mesomorphic Property Relationships. Molecular structure of organic compounds and their liquid crystalline properties are closely related. Table 1 summarizes the transition temperatures, mesomorphic behavior, and molecular structures of 8FBAA, 8ABFA, and structurally related compounds reported in the literature $[19,26-30]$.

Halogen groups at the terminal position showed strong influence on the mesomorphic properties of a molecule as indicated by the current work (Table 1). It can be seen that the transitions temperatures of $8 \mathrm{ABFA}$ are lower than those of $8 \mathrm{BACl}, 8 \mathrm{BABr}$, and $8 \mathrm{BAI}$. Size of fluorosubstituent is smaller than the chloro-, bromo-, and iodosubstituents and therefore less easily polarized due to the electrons on this atom which are tightly held and located closer to the nucleus [31]. Lower molecular polarizability attributed to the smaller size of fluoro substituent decreased the phase stability in 8ABFA [15]. This phenomenon is supported by the similar characteristic 


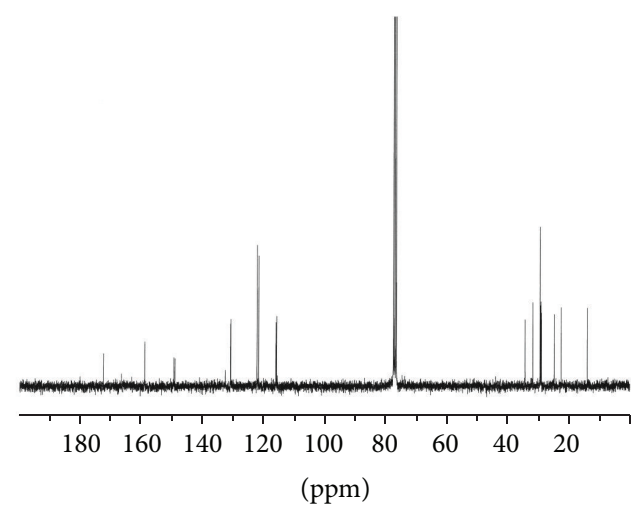

(a)

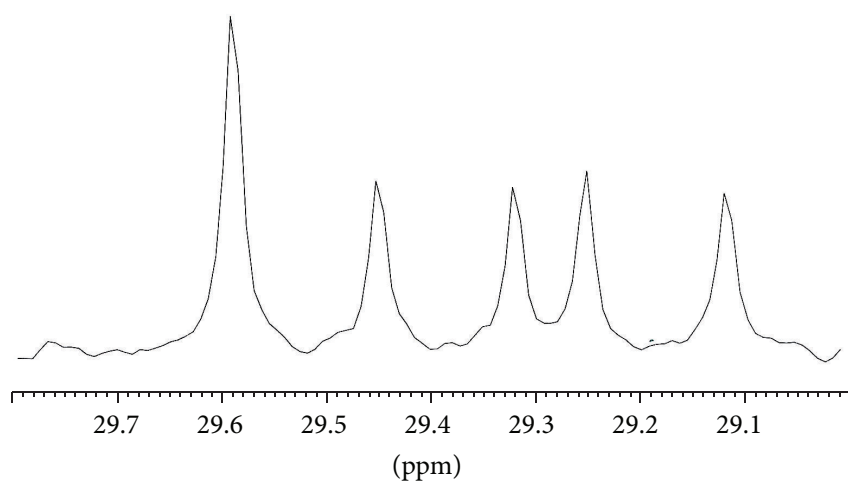

(b)

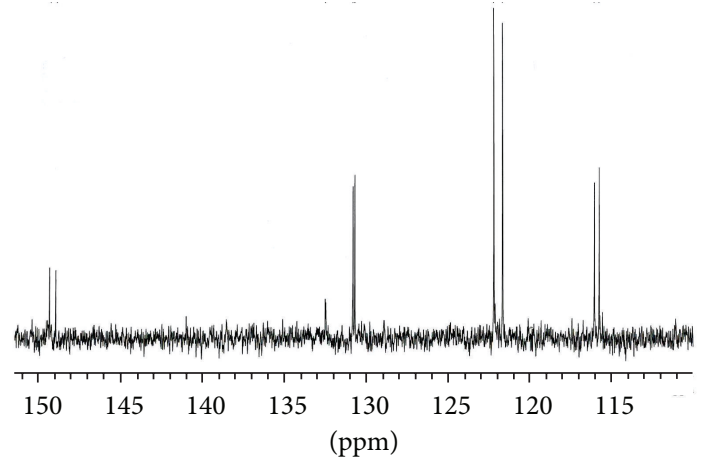

(c)

FIGURE 3: ${ }^{13} \mathrm{C}$ NMR spectrum of 12FBAA (a) full spectrum, (b) expanded spectrum for aliphatic region, and (c) expanded spectrum for aromatic region.

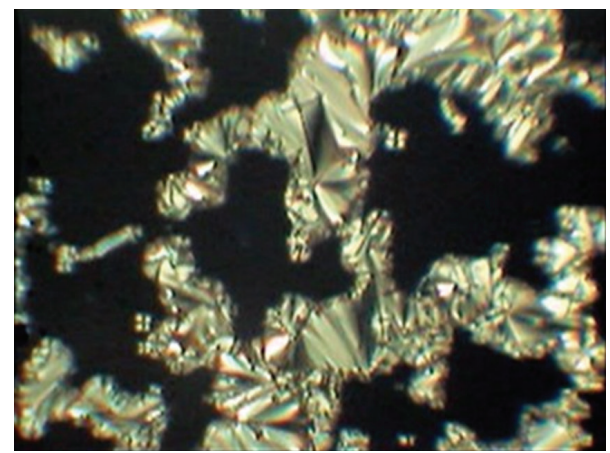

FIGURE 4: Optical photomicrograph of 12ABFA showing the coexistence of fan-shaped and homeotropic textures. It showed characteristics of SmA phase.

observed for $\mathrm{X}-\mathrm{AB}$ and $\mathrm{X}-\mathrm{ABOH}$ series (where $\mathrm{X}=\mathrm{F}, \mathrm{Cl}, \mathrm{Br}$, I) wherein the melting and transition temperatures ascended as the size of halogen group increased.

Secondly, polarisability of halogen terminal groups can also control the phase stability of liquid crystal molecules. Compound having lower polarisability halogen group (8ABFA and $8 \mathrm{BACl}$ ) possessed less stable monotropic smectic phase than $8 \mathrm{BABr}$ and $8 \mathrm{BAI}$ which exhibited enantiotropic smectic phase. Similar pattern was also reported for $\mathrm{X}-\mathrm{AB}$ series whereby $\mathrm{F}-\mathrm{AB}$ (monotropic phase) possessed

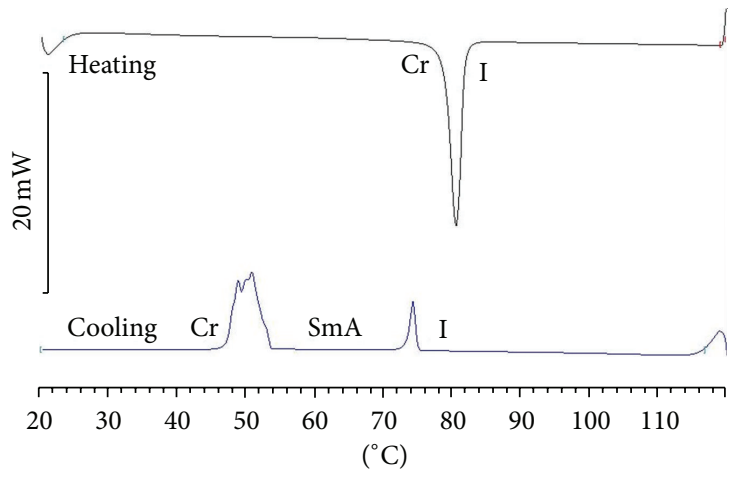

FIGURE 5: DSC thermogram of 12ABFA.

lower phase stability than $\mathrm{Cl}-\mathrm{AB}$ and $\mathrm{Br}-\mathrm{AB}$ (enantiotropic phase).

Difference in the linking groups (ester group in $8 \mathrm{ABFA}$ and ether group in F-AB) between the phenyl ring and the alkyl chain can also cause change in the mesomorphic properties. The ether linking group provides greater linearity to molecules rather than the ester group, thus resulting in the wider phase range being observed in $\mathrm{F}-\mathrm{AB}$ when compared to 8ABFA. In addition, 8ABFA possessed higher melting point than $\mathrm{F}-\mathrm{AB}$ owing to the $\pi$-electrons associated with 
the carbonyl group which provides greater intermolecular interactions among molecules [32].

Influence of lateral hydroxyl group was relatively apparent on the mesomorphic properties of Schiff base. Intramolecular hydrogen bonding between ortho-hydroxyl group and nitrogen atom has increased the molecular polarisability, and therefore compounds with hydroxyl group (X-ABOH series) possessed higher melting and clearing temperatures than their analogous compounds without hydroxyl group. However, the increased polarisability at the lateral of a molecule caused depression of $\mathrm{SmB}$ arrangement and thus impeding $\mathrm{SmB}$ formation in the $\mathrm{X}-\mathrm{ABOH}$ series.

The position of the imine linking group defines the direction of the carbonyl group. The different directions of the carboxyl groups between the phenyl and alkyl units cause remarkable changes on the dipole moment as that reported for some banana-shaped mesogens [33]. The smectic properties of the molecules are strongly affected by not only the electrostatic interactions but also the geometrical circumstances [34]. Furthermore, $n$ FBAA series possessed higher melting point compared to $n \mathrm{ABFA}$ having the same number of carbons $(n)$ at the alkyl chain and subsequently impeded the mesophase formation. This suggests that the reversed imine linkage can also depress the thermal stability of a compound ( $n$ FBAA).

\section{Conflict of Interests}

The authors do not have a direct financial relation with the commercial identities mentioned in the paper including Finnigan, Perkin Elmer, Bruker, Merck, Mettler Toledo, Carl Zeiss and Linkam that might lead to a conflict of interest for any of the authors.

\section{Acknowledgments}

The authors would like to thank Universiti Tunku Abdul Rahman and the Ministry of Higher Education of Malaysia for the financial supports and research facilities.

\section{References}

[1] L. Petti, M. Rippa, A. Fiore, L. Manna, and P. Mormile, "Optically induced light modulation in an hybrid nanocomposite system of inorganic CdSe/CdS nanorods and nematic liquid crystals," Optical Materials, vol. 32, pp. 1011-1016, 2010.

[2] N. A. Shurpo, M. S. Vakshtein, and N. V. Kamanina, "Effect of CdSe/ZnS semiconductor quantum dots on the dynamic properties of nematic liquid-crystalline medium," Technical Physics Letters, vol. 36, no. 4, pp. 319-321, 2010.

[3] M. H. Hoang, M. J. Cho, K. H. Kim, T. W. Lee, J.-I. Jin, and D. H. Choi, "Semiconducting 2,3,6,7,10,11-Hexakis $\{[4-$ (5-dodecylthiophen-2-yl)phenyl] ethynyl\} triphenylene and its discotic liquid crystalline properties," Chemistry Letters, vol. 39, no. 4, pp. 396-397, 2010.

[4] F. Yuksel, D. Atilla, and V. Ahsen, "Synthesis and characterization of liquid crystalline unsymmetrically substituted phthalocyanines," Polyhedron, vol. 26, no. 15, pp. 4551-4556, 2007.
[5] B. Y. Zhang, F. B. Meng, M. Tian, and W. Q. Xiao, "Side-chain liquid-crystalline polysiloxanes containing ionic mesogens and cholesterol ester groups," Reactive and Functional Polymers, vol. 66, pp. 551-558, 2005.

[6] P. J. Collings and M. Hird, Introduction to Liquid Crystals Chemistry and Physics, Taylor \& Francis, London, UK, 1998.

[7] S. Singh and D. A. Dunmur, Liquid Crystals: Fundamentals, World Scientific, London, UK, 2002.

[8] H. Kelker and B. Scheurle, "A liquid crystalline (nematic) phase with a particularly low solidification point," Angewandte Chemie International Edition, vol. 8, pp. 884-885, 1969.

[9] B. B. Eran, A. Nesrullajev, and N. Y. Canli, "Characterization and investigation of the mesogenic, thermo-morphologic and thermotropic properties of new chiral $(S)$-5-octyloxy-2-[\{4-(2methylbuthoxy)-phenylimino(methyl]phenol liquid crystalline compound," Materials Chemistry and Physics, vol. 111, no. 2-3, pp. 555-558, 2008.

[10] S. T. Ha, L. K. Ong, S. T. Ong et al., "Synthesis and mesomorphic properties of new Schiff base esters with different alkyl chains," Chinese Chemical Letters, vol. 20, no. 7, pp. 767-770, 2009.

[11] S. T. Ha, G. Y. Yeap, and P. L. Boey, "Synthesis and liquid crystalline properties of new schiff bases $\mathrm{N}$-[4-(4-nalkanoyloxybenzoyloxy)benzylidene]-4-cyano-, 4-hydroxy-, 4thio-and 4-nitroanilines," Australian Journal of Basic \& Applied Sciences, vol. 3, no. 4, pp. 3417-3422, 2009.

[12] M. Parra, J. Vergara, C. Zuniga, E. Soto, T. Sierra, and J. L. Serrano, "New chiral Schiff's bases with a 1,3,4-thiadiazole ring in the mesogenic core: synthesis, mesomorphic and ferroelectric properties," Liquid Crystals, vol. 32, no. 4457, 462 pages, 2004.

[13] A. K. Prajapati and M. C. Varia, "Azomesogens with polar chloro, nitro and phenolic -OH substituents," Liquid Crystals, vol. 35, no. 11, pp. 1271-1277, 2008.

[14] R. Vora, A. K. Prajapati, and J. Kevat, "Effect of terminal branching on mesomorphism," Molecular Crystals and Liquid Crystals, vol. 357, no. 1, pp. 229-237, 2001.

[15] G.-Y. Yeap, S.-T. Ha, P.-L. Lim et al., "Synthesis and mesomorphic properties of schiff base esters ortwo-hydroxypara-alkyloxybenzylidene-para-substituted anilines," Molecular Crystals and Liquid Crystals, vol. 423, pp. 73-84, 2004.

[16] G. Y. Yeap, S. T. Ha, P. L. Boey, W. A. K. Mahmood, M. M. Ito, and Y. Youhei, "Synthesis and characterization of some new mesogenic schief base esters N-[4-(4-n-hexadecanoyloxybenzoyloxy)-benzylidene]-4-substituted anilines," Molecular Crystals and Liquid Crystals, vol. 452, no. 1, pp. 73-90, 2006.

[17] G.-Y. Yeap, S.-T. Ha, P.-L. Lim et al., "Nematic and smectic a phases in ortho-hydroxy-para-hexadecanoyloxbenzylidenepara-substituted anilines," Molecular Crystals and Liquid Crystals, vol. 452, no. 1, pp. 63-72, 2006.

[18] G.-Y. Yeap, S.-T. Ha, P.-L. Lim et al., "Synthesis, physical and mesomorphic properties of Schiff's base esters containing ortho-, meta- and para-substituents in benzylidene- $4^{\prime}$ alkanoyloxyanilines," Liquid Crystals, vol. 33, no. 2, pp. 205-211, 2006.

[19] Z. Galewski, "Molecular crystals and liquid crystals science and technology. Section A. molecular crystals and liquid crystals," Molecular Crystals and Liquid Crystals, vol. 249, pp. 43-49, 1994.

[20] Z. Galewski and H. J. Coles, "Liquid crystalline properties and phase situations in 4-chlorobenzylidene-4'-alkylanilines," Journal of Molecular Liquids, vol. 79, no. 1, pp. 77-87, 1999.

[21] S. Sakagami and M. Nakamizo, "Liquid crystalline properties of $\mathrm{N}$-(4-alkoxybenzylidene)-4-halogenoanilines," Bulletin of the Chemical Society of Japan, vol. 53, no. 1265, 266 pages, 1980. 
[22] J. S. Dave and M. Menon, "Azomesogens with a heterocyclic moiety," Bulletin of Material Science, vol. 23, pp. 237-238, 2000.

[23] V. F. Petrov, M. Duan, H. Okamoto, J. Mu, Y. Shimizu, and S. Takenaka, "Halogenation in achiral liquid crystals: terminal and linking substitutions," Liquid Crystals, vol. 28, no. 3, pp. 387-410, 2001.

[24] H. Sackmann and D. Demus, "The polymorphism of liquid crystals," Molecular Crystals and Liquid Crystals, vol. 2, pp. 81102, 1966.

[25] G. W. Gray and J. W. Goodby, Smectic Liquid Crystals: Textures and Structures, Leonard Hill, London, UK, 1984.

[26] C. Gandolfo, D. Grasso, G. Buemi, and G. Torquati, "Phase transitions and structural studies of p-n-alkoxybenzyliden- $p^{\prime}$ fluoro-anilines," Il Nuovo Cimento D, vol. 10, no. 11, pp. 1363$1371,1988$.

[27] S. Sakagami, T. Koga, and A. Takase, "Liquid crystalline properties and photochromism of 4-halogeno-N-(4-alkoxysalicylidene) anilines," Molecular Crystals and Liquid Crystals, vol. 373, no. 1, pp. 71-81, 2002.

[28] S.-T. Ha, L.-K. Ong, Y. Sivasothy et al., "Mesogenic schiff base esters with terminal chloro group: synthesis, thermotropic properties and X-ray diffraction studies," International Journal of Physical Sciences, vol. 5, no. 5, pp. 564-575, 2010.

[29] S. T. Ha, T. L. Lee, H. C. Lin et al., "Mesogenic Schiff base with bromo end group: synthesis and thermotropic properties," Asian Journal of Chemistry, vol. 24, no. 8, pp. 3679-3684, 2012.

[30] S.-T. Ha, T.-L. Lee, S.-L. Lee, S. Sreehari Sastry, and Y.-F. Win, "Schiff base liquid crystals with terminal iodo group: synthesis and thermotropic properties," Scientific Research and Essays, vol. 6, no. 23, pp. 5025-5035, 2011.

[31] T. W. G. Solomons, Fundamentals of Organic Chemistry, John Wiley \& Sons, New York, NY, USA, 1994.

[32] S. Kumar, Liquid Crystals: Experimental Study of Physical Properties and Phase Transitions, Cambridge University Press, Cambridge, UK, 2001.

[33] S. A. R. Krishnan, W. Weissflog, G. Pelzl et al., "DFT and MD studies on the influence of the orientation of ester linkage groups in banana-shaped mesogens," Physical Chemistry Chemical Physics, vol. 8, no. 10, pp. 1170-1177, 2006.

[34] Y. Sakurai, S. Takenaka, H. Miyake, H. Morita, and T. Ikemoto, "Molecular structure and smectic properties. Part 1. The effect of linkages on smectic A thermal stability in three aromatic ring compounds linked by ester groups," Journal of the Chemical Society, Perkin Transactions 2, no. 9, pp. 1199-1204, 1989. 

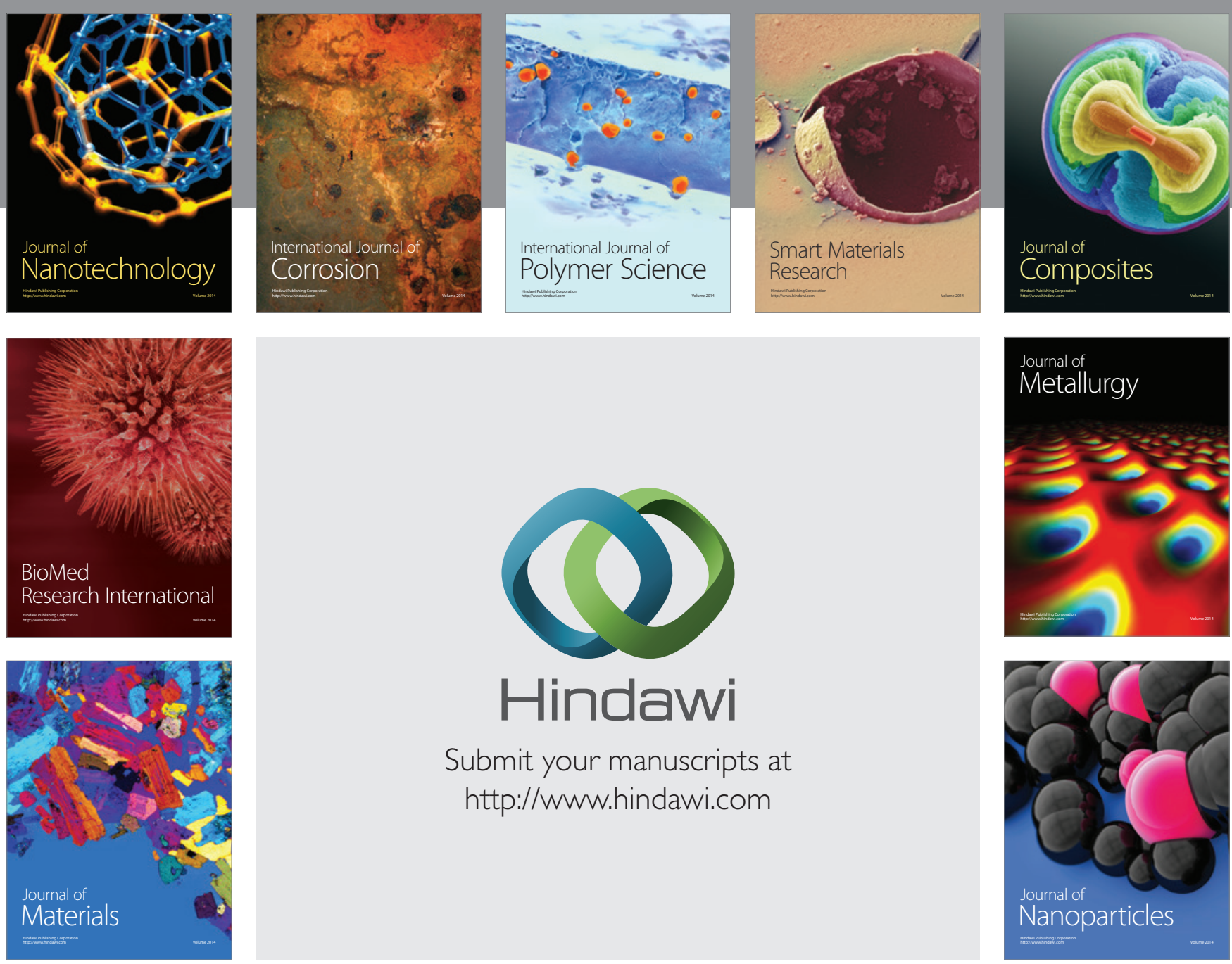

Submit your manuscripts at http://www.hindawi.com
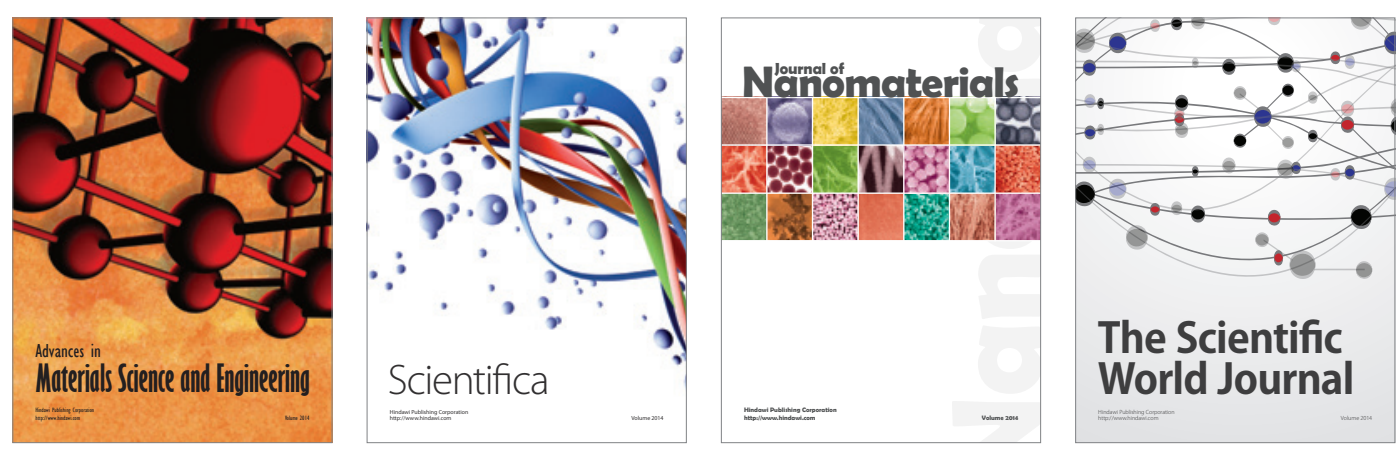

\section{The Scientific World Journal}
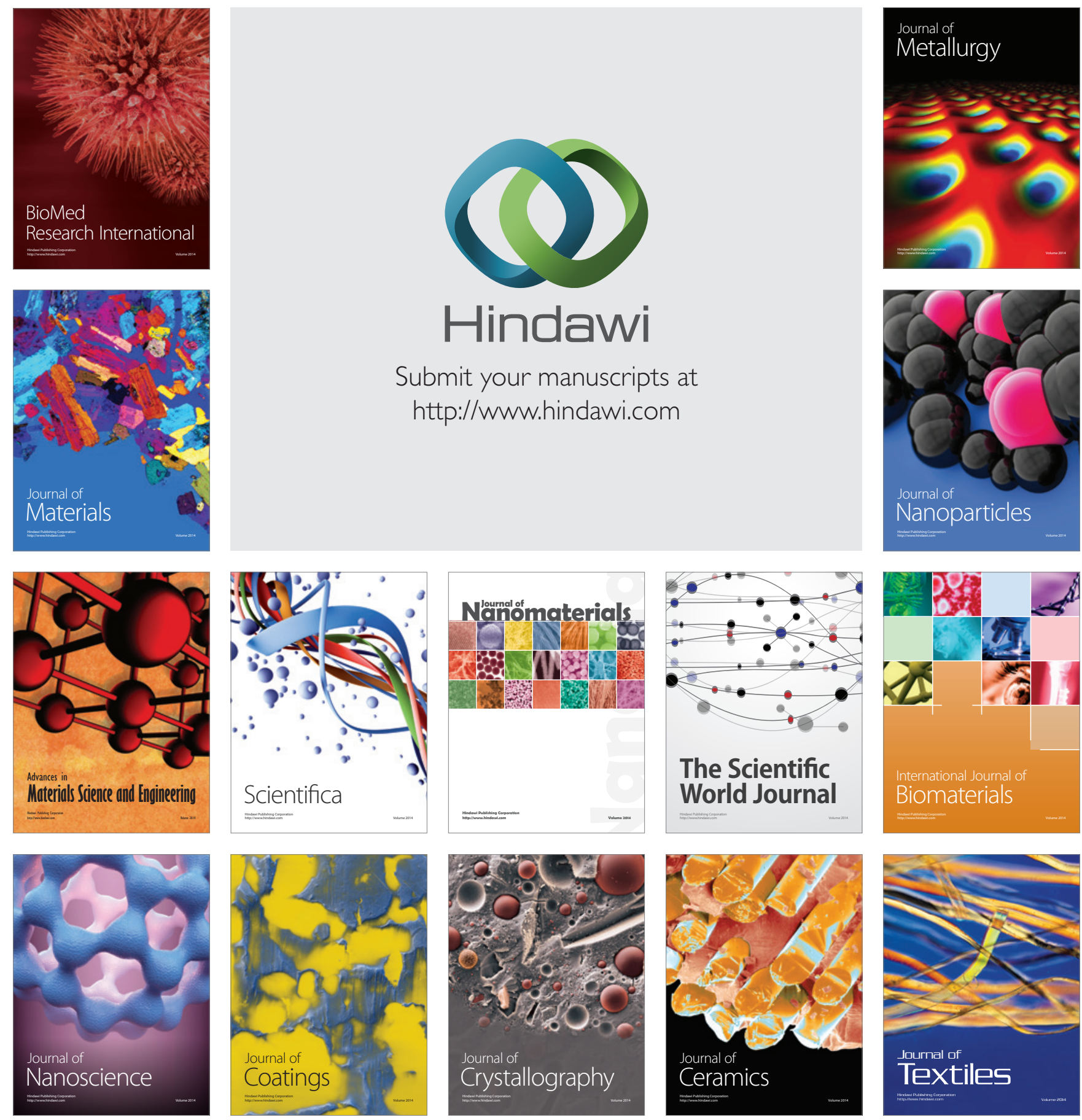